\title{
The impact of corporate social responsibility for employees to organisational commitment
}

\author{
Tien D. Mai ${ }^{1 *}$, Thuy T. T. Nguyen ${ }^{2}, \&$ Tinh Nguyen ${ }^{3}$ \\ ${ }^{1}$ Faculty of Fisheries, Nong Lam University, Ho Chi Minh City, Vietnam \\ ${ }^{2}$ School of Tourism, University of Economics Ho Chi Minh City, Ho Chi Minh City, Vietnam \\ ${ }^{3}$ Center for Vietnamese Technology Research and Development
}

\author{
ARTICLE INFO \\ Research Paper \\ Received: November 28, 2018 \\ Revised: April 20, 2019 \\ Accepted: August 08, 2019 \\ Keywords \\ Employees \\ Organizational commitment \\ Social responsibility

\section{${ }^{*}$ Corresponding author} \\ Mai Dang Tien \\ Email: tien.maidang@hcmuaf.edu.vn
}

\begin{abstract}
This study explores the factors of corporate social responsibility (CSR) for employees affecting organizational commitment, and then measures the impact of CSR for employees to organizational commitment. The study was conducted by surveying 200 employees working for companies in Ho Chi Minh City. The results of the correlation analysis show that CSR for employees influences the organizational commitment. In addition, the regression analysis shows that more specific results such as job autonomy, benefits (the most influential factor) have positive impacts on "affective commitment" of Vietnamese employees. For "continuance commitment", besides job autonomy, benefits, the factor "training and development" also shows a positive influence. Meanwhile, the factors related to health and safety, the balance between job and life are not as effective as those in other countries.
\end{abstract}

Cited as: Mai, T. D., Nguyen, T. T. T., \& Nguyen, T. (2020). The impact of corporate social responsibility for employees to organisational commitment. The Journal of Agriculture and Development 19(1), 1-8. 


\title{
Ảnh hưởng của trách nhiệm xã hội của doanh nghiệp đối vởi người lao động đến cam kết tổ chức
}

\author{
Mai Đăng Tiến ${ }^{1 *}$, Nguyễn Thị Thu Thủy ${ }^{2}$ \& Nguyễn Tính ${ }^{3}$ \\ ${ }^{1}$ Khoa Thủy Sản, Trường Đại Học Nông Lâm TP.HCM, TP. Hồ Chí Minh \\ ${ }^{2}$ Viện Du Lịch, Trường Đại Học Kinh Tế TP.HCM, TP. Hồ Chí Minh \\ ${ }^{3}$ Trung Tâm Nghiên Cứu và Phát Triển Công Nghệ Việt
}

\author{
THÔNG TIN BÀI BÁO \\ Bài báo khoa học \\ Ngày nhận: 28/11/2018 \\ Ngày chỉnh sưa: 20/04/2019 \\ Ngày chấp nhận: 08/08/2019 \\ Từ khóa \\ Cam kết tổ chức \\ Người lao động \\ Trách nhiệm xã hội \\ *Tác giả liên hệ \\ Mai Đăng Tiến \\ Email: tien.maidang@hcmuaf.edu.vn
}

\section{TÓM TẮT}

Nghiên cứu này tìm hiểu các nhân tố thuộc về trách nhiệm xã hội của doanh nghiệp (Corporate Social Responsibility - CSR) đối với người lao động ảnh hưởng đến cam kết tổ chức, sau đó đo lường tác động của CSR đối với người lao động đến cam kết tổ chức. Nghiên cứu được tiến hành bằng cách điều tra khảo sát 200 người lao động đang làm việc trong các doanh nghiệp trên địa bàn TP.HCM. Kết quả phân tích tương quan cho thấy CSR đối với người lao động có ảnh hưởng đến "cam kết tổ chức". Bên cạnh đó, phân tích hồi quy cho thấy những kết quả cụ thể hơn như sự tự chủ trong công việc, lợi ích nhận được có ảnh hưởng đến tích cực (trong đó có yếu tố lợi ích tác động mạnh nhất) đến "cam kết tình cảm" của người lao động Việt Nam. Đối với "cam kết duy trì", ngoài sự tự chủ trong công việc cũng như lợi ích nhận được thì yếu tố "đào tạo và phát triển" cũng cho thấy tác động tích cực. Trong khi đó, các yếu tố liên quan đến sức khỏe, an toàn lao động, sự cân bằng giữa công việc và cuộc sống lại không thể hiện được tầm ảnh hưởng như ở một số nước khác.

\section{1. Đặt Vấn Đề}

Nguồn nhân lực đóng vai trò quan trọng trong quyết định sức mạnh cạnh tranh cho doanh nghiệp bởi nó tác động trực tiếp đến hoạt động thiết lập và thực thi chiến lược kinh doanh của tổ chức. Tuy nhiên, một số doanh nghiệp gần đây đối mặt với thực trạng là tỷ lệ nhân viên giỏi xin thôi việc đang có xu hướng gia tăng. Thực trạng này kéo dài sẽ làm giảm sức mạnh cạnh tranh của doanh nghiệp nghiêm trọng, bởi khi nguồn tài sản nhân lực bị mất đi thì doanh nghiệp mất đi nguồn chất xám to lớn, doanh nghiệp sẽ không thể đề ra và thực hiện chiến lược kinh doanh hiệu quả và việc kinh doanh thất bại là hậu quả tất yếu.

Người tài trong doanh nghiệp rất hiếm. Để giữ được nhân viên giỏi, các nhà quản trị nhân lực đã áp dụng nhiều cách thức, tuy nhiên phần lớn chú tâm vào các yếu tố vật chất. Lẽ dĩ nhiên, một mức lương cạnh tranh trên thị trường là điều cần thiết, tuy nhiên cần lưu ý là tiền không phải công cự hiệu quả nhất để giữ nhân viên và khiến họ thật sự gắn bó với doanh nghiệp. Lãnh đạo doanh nghiệp cần nỗ lực nhiều hơn để giữ nhân viên và phát triển tài năng của họ. Phương thức dùng các giá trị thuộc về trách nhiệm xã hội của doanh nghiệp (Corporate Social Responsibility-CSR) là một "vũ khí" mới rất đáng quan tâm trong "cuộc chiến giành nguồn nhân lực".

Trách nhiệm xã hội của doanh nghiệp là khái niệm rộng lớn, thách thức về đo lường. Chẳng hạn, Carroll (1979) đã đưa ra mô hình CSR như một cách thức để nâng cao hiệu quả trong công ty. Carroll (1999) đã tổng hợp khá nhiều nghiên cứu định nghĩa, giải thích về CSR trong suốt quá trình hình thành và phát triển của nó đồng thời đề ra kỳ vọng những bước tiến trong tương lai trong việc nghiên cứu vấn đề này. Brammer \& ctv. (2007) đã điều tra mối quan hệ giữa cam kết tổ chức và nhận thức của nhân viên về CSR trong một mô hình dựa trên lý thuyết bản sắc xã hội, 
nhấn mạnh tầm quan trọng của sự khác biệt về giới tính và cho rằng CSR liên quan tích cực đến cam kết tổ chức... Trách nhiệm xã hội của doanh nghiệp được chứng minh là một trong những cách thức tối ưu nhất để giữ chân nhân viên giỏi cho doanh nghiệp bởi nó hướng con người làm việc vì những mục đích cao đẹp. Nhân viên giỏi hài lòng với phương thức CSR bởi nó làm thỏa mãn những nhu cầu làm việc vì giá trị xã hội của họ. Những nghiên cứu tiến hành trong quá khứ cho thấy rằng, CSR sẽ tăng mức độ cam kết của các nhân viên đối với tổ chức bởi vì nó quan tâm về phúc lợi của người lao động cũng như gia đình của họ (Ali \& ctv., 2010), đóng góp của CSR cho cộng đồng sẽ làm cho nhân viên tăng động lực trong thực hiện nhiệm vụ của họ, vì họ có thể cảm thấy tự hào khi làm việc trong một tổ chức quan tâm đến toàn thể xã hội. Bên cạnh đó, họ cam kết hơn với tổ chức khi họ cảm thấy rằng, họ là một trong các thành viên có đóng góp tích cực cho xã hội.

Tuy nhiên, đây vẫn còn là một vấn đề khá mới mẻ ở Việt Nam, thời gian gần đây cũng có một số bài báo đăng tải những nghiên cứu về CSR tại Việt Nam. Bài viết của Le (2006) về CSR trong vấn đề tiền lương đề cập tới vai trò của tiền lương như các mức lương vừa thể hiện vị trí công việc, vừa thể hiện sự chia sẻ lợi ích giữa các tổ chức, các doanh nghiệp và người lao động, vừa thể hiện sự phát triển nghề nghiệp của mỗi cá nhân người lao động. Nguyen \& Luu (2008) bàn luận về CSR thông qua những khái niệm, nghiên cứu trước đó, tình hình thực hiện CSR tại Việt Nam và đề ra những kiến nghị đổi mới trong tư duy quản lý của Nhà nước. Nguyen (2010) đã nêu lên những lợi ích của việc thực hiện CSR và gợi ý về việc lồng ghép CSR vào việc quản trị nguồn nhân lực. Pham (2013) nghiên cứu chỉ số CSR ứng dụng tại Công ty Cổ phần Dệt may 23/9. Mặc dù nhiều tổ chức quan tâm nhưng những nghiên cứu trước đây chủ yếu bàn luận về CSR nói chung, vẫn còn thiếu các nghiên cứu tập trung về vấn đề tác động của CSR đối với người lao động đến cam kêt tổ chức. Vì vậy, mục tiêu nghiên cứu này là tập trung vào xác định các nhân tố thuộc về CSR đối với người lao động có ảnh hưởng đến cam kết tổ chức và đo lường tác động này.

\section{Phương Pháp Nghiên Cứu}

Ảnh hưởng của CSR đối với cam kết tổ chức đã được chứng minh trong rất nhiều nghiên cứu.
Trong nghiên cứu này, tác giả tham khảo một số nghiên cứu của Mathieu \& Zajac (1990), Meyer \& Allen (1991), Meyer \& Allen (1997), Agarwal \& Ferratt (1999), Chang (1999), Meyer \& ctv. (2004), Dockel \& ctv. (2006), Jaros (2007). Trong đó, mô hình nghiên cứu đề xuất (Hình 1 ) và thang đo được xây dựng chủ yếu dựa trên nghiên cứu của Dockel \& ctv. (2006) và Jaros (2007).

Sau khi xây dựng các khái niệm nghiên cứu, các giả thuyết nghiên cứu và mô hình nghiên cứu tiến hành thực hiện qua hai bước nghiên cứu sơ bộ và chính thức. Nghiên cứu sơ bộ gồm một nghiên cứu định tính sơ bộ thực hiện theo phương pháp thảo luận tay đôi, phỏng vấn các chuyên gia để hiệu chỉnh lại thang đo trước khi tiến hành khảo sát sơ bộ. Tác giả xây dựng dàn bài thảo luận, sau đó gặp gỡ lần lượt từng chuyên gia phỏng vấn, ghi nhận lại những ý kiến, điều chỉnh thang đo, sau đó tiếp tục phỏng vấn người tiếp theo cho đến khi không còn ý kiến nào khác biệt. Một nghiên cứu định lượng sơ bộ thực hiện qua khảo sát, thu thập dữ liệu sơ bộ từ 20 nhân viên, phân tích để đánh giá và điều chỉnh thang đo, từ đó xây dựng thang đo chính thức.

Nghiên cứu định lượng chính thức được tiến hành khi bảng câu hỏi được chỉnh sửa từ kêt quả nghiên cứu sơ bộ, nghiên cứu này khảo sát trực tiếp 250 nhân viên đang làm việc trong các công ty tại địa bàn TP.HCM (thuộc lĩnh vực quốc doanh, ngoài quốc doanh và công ty có vốn đầu tư nước ngoài) theo phương pháp lấy mẫu thuận tiện. Sau khi có kết quả khảo sát tiến hành phân tích dữ liệu để đánh giá thang đo, kiểm định các giả thuyết và mô hình nghiên cứu với sự hỗ trợ của phần mềm SPSS.

Dữ liệu được tiến hành xử lý qua các bước: kiểm định thang đo bằng hệ số tin cậy Cronbach Alpha, những nhân tố nào không đạt yêu cầu về độ tin cậy (Cronbach's Alpha < 0,6) sẽ bi loại. Tất cả các biến quan sát của những nhân tố đạt độ tin cậy sẽ tiếp tục được phân tích nhân tố khám phá (EFA) để đánh giá lại mức độ hội tụ của các biến quan sát theo các nhân tố trong mô hình đề xuất ban đầu, nếu có phát sinh tăng, giảm các nhân tố sẽ điều chỉnh lại để có mô hình nghiên cứu chính thức. Cuối cùng phân tích tác động của CSR đối với người lao động đến cam kết tổ chức bằng mô hình hồi quy đa biến. Trước tiên ta xem xét ma trận hệ số tương quan để nhận định về mối tương quan tuyến tính giữa các biến. Sau đó đánh giá độ phù hợp của mô hình hồi quy thông qua hệ số xác định $\mathrm{R}^{2}$ hiệu chỉnh và kiểm định 


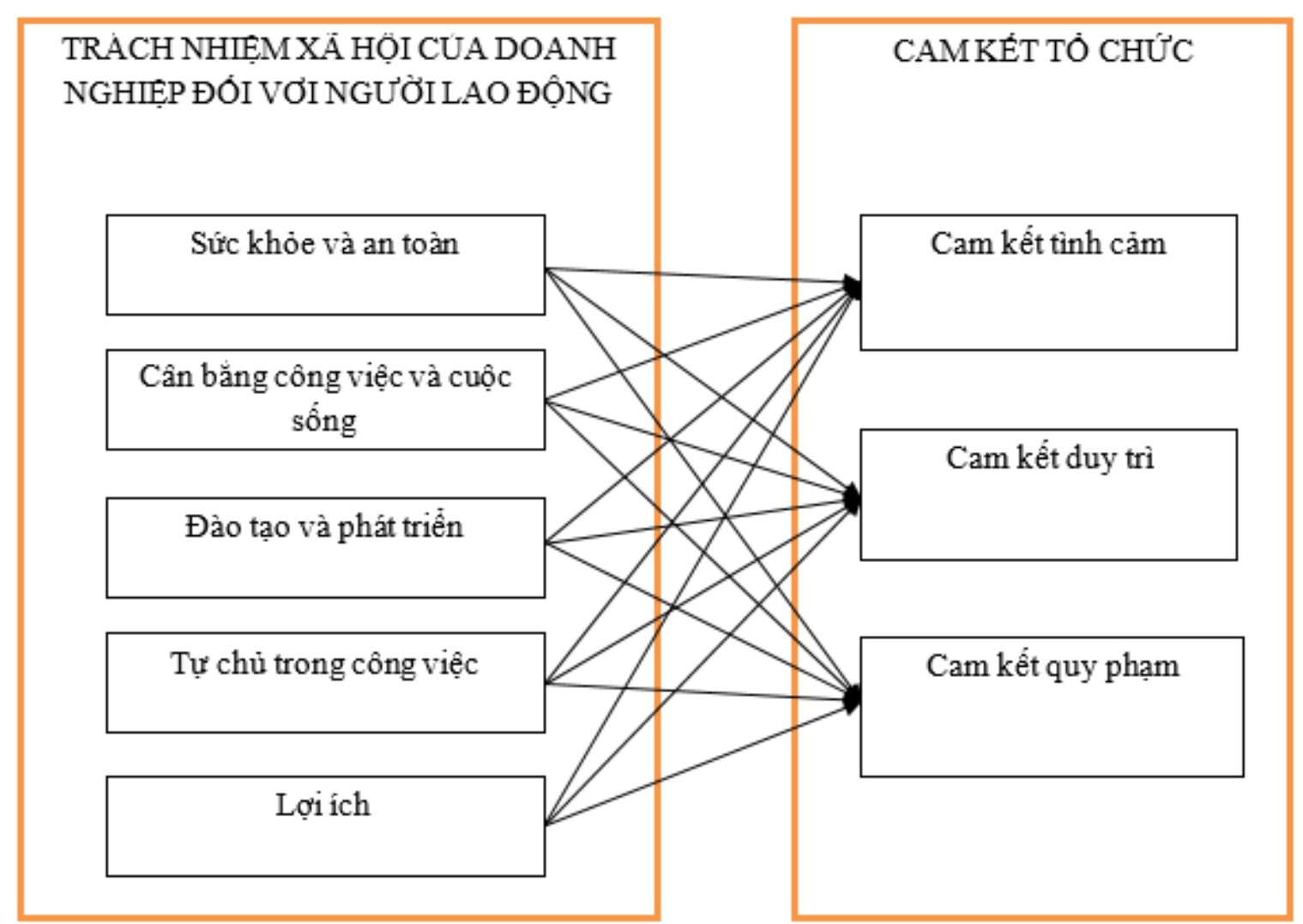

Hình 1. Mô hình nghiên cứu đề xuất.

độ phù hợp của mô hình bằng kiểm định $\mathrm{F}$, xem xét ý nghĩa các hệ số hồi qui riêng phần trong mô hình. Đồng thời, kiểm tra có xảy ra hiện tượng đa cộng tuyến (thông qua hệ số phóng đại phương sai - VIF), hiện tượng tự tương quan (thông qua hệ số Durbin-Waston) và hiện tượng phương sai sai số thay đổi (sử dụng kiểm định tương quan hạng Spearman giữa phần dư chuẩn hóa với các biến độc lập) hay không.

\section{Kết Quả và Thảo Luận}

Tỷ lệ mẫu điều tra khá đồng đề về mặt giới tính (46\% nam và $54 \%$ nữ). Đối tượng điều tra phần lớn là những nhân viên trẻ (dưới 30 tuổi chiếm đến $69 \%$ ), theo tác giả đánh giá đây là một điểm tích cực vì người trẻ hiện nay có những yêu cầu cao hơn trong công việc. Xét về mặt kinh nghiệm làm việc, đối tượng điều tra chủ yếu làm việc từ một đến ba năm (44\%), thời gian này cũng đủ để nhân viên có thể đánh giá về công ty mình đang làm việc, tiếp sau là những đối tượng đã gắn bó với công ty trên 10 năm (21\%) cho thấy họ đã có sự cam kết cao với công ty và thông qua phân tích chúng ta có thể đánh giá được những nhân tố thuộc CSR đối với người lao động tác động như thế nào đến cam kết tổ chức.

Quá trình kiểm tra độ tin cậy của thang đo bằng hệ số Cronbach's Alpha cho kết quả thang đo CSR đối với người lao động các biến thành phần đều được giữ nguyên như ban đầu, riêng thành phần "tự chủ trong công việc" từ 5 biến quan sát ban đầu loại bỏ 1 biến. Tiếp tục phân tích nhân tố khám phá EFA để đánh giá lại mức độ hội tụ các biến quan sát theo các nhân tố trong mô hình đề xuất ban đầu, các biến có trọng số nhỏ hơn 0,5 và các biến có hệ số tải nhân tố không đạt độ phân biệt cao giữa các nhân tố (nhỏ hơn $0,3)$ sẽ bị loại. Với cách thức này sau 3 lần phân tích (do phân tích lần 1 và lần 2 có các biến không đạt yêu cầu, tác giả quyết định loại bỏ và tiếp tục thử nghiệm cho lần chạy tiếp theo) thì 5 nhân tố ban đầu của CSR đối với người lao động vẫn được giữ tuy số lượng biến quan sát giảm từ 25 xuống 


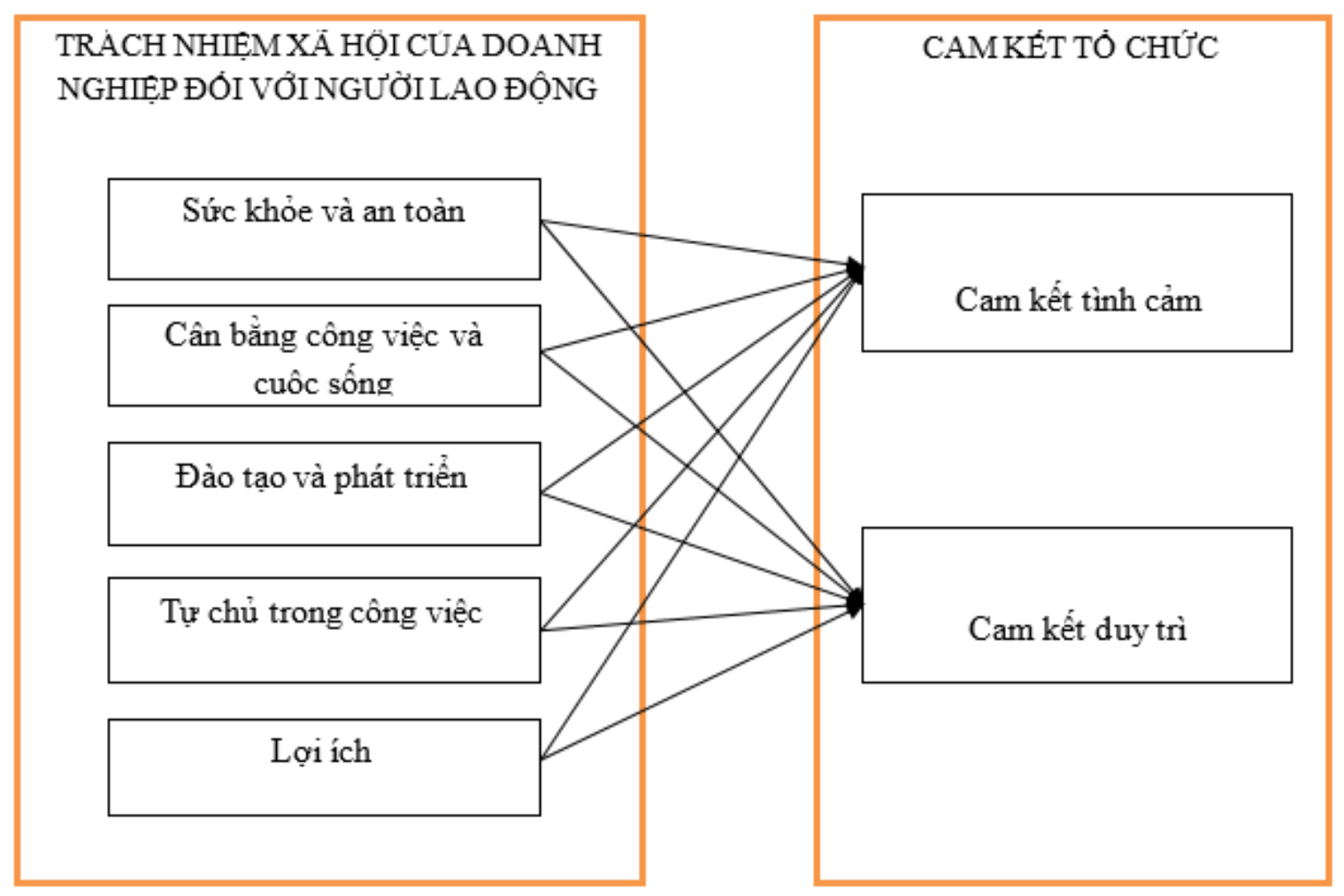

Hình 2. Mô hình nghiên cứu chính thức.

19 biết và giải thích được $73,47 \%$ biến thiên của dữ liệu.

Thực hiện tương tự cho thang đo cam kết tổ chức thì kết quả kiểm định độ tin cậy của thang đo bằng hệ số Cronbach's Alpha cho thấy tất cả các thành phần đều đạt yêu cầu. Tiếp tục phân tích nhân tố khám phá EFA. Và cụng đến lần phân tích thứ 3 (sau khi loại bỏ các biến không đạt yêu cầu ở lần phân tích thứ nhất và thứ hai) từ 3 nhân tố như giả thuyết ban đầu rút ra được 2 nhân tố mới, các biến thuộc nhân tố "cam kết tình cảm" và "cam kết quy phạm" từ giả thuyết ban đầu nhóm chung thành một nhóm. Mô hình nghiên cứu được điều chỉnh lại như Hình 2.

Kết quả kiểm định độ tin cậy Cronbach Alpha các thang đo (Bảng 1) cho thấy tất cả thang đo các nhân tố trong mô hình nghiên cứu chính thức đều đạt yêu cầu về độ tin cậy (Cronbach Alpha trên 0,6). Các hệ số tương quan biến-tổng của các thang đo đều đạt yêu cầu (trên 0,3 ) nên tất cả thang đo được chấp nhận đưa vào phân tích.

Kết quả kiểm định tương quan giữa các biến và phân tích hồi quy tuyến tính tác động của CSR đối với người lao động đến "cam kết tình cảm" (Bảng 2) cho thấy mô hình được xây dựng phù hợp (múc ý nghĩa $P<0,05$ ). Mô hình cũng không bị hiện tượng tự tương quan (hệ số DurbinWatson nằm trong khoảng từ 1 đến 3 ) (Ho, 2009), không xảy ra hiện tượng đa cộng tuyến (Hệ số phóng đại phương sai $\mathrm{VIF}<3$ ) và không vi phạm giả định phương sai không thay đổi (kết quả phân tích tương quan hạng Spearman giữa phần dư chuẩn hóa với các biến độc lập đều có giá trị $P$ $>0,05)$. Trong 5 nhân tố đo lường "cam kết tình cảm" như mô hình có 02 nhân tố ảnh hưởng đến "cam kết tình cảm" đó là "tự chủ trong công việc" và "lợi ích" (mức ý nghĩa $P<0,05$ ). Hệ số hồi quy mang dấu dương thể hiện các yếu tố trong mô hình hồi quy trên ảnh hưởng thuận chiều đến "cam kết tình cảm" của nhân viên.

Như vậy trong 5 nhân tố đưa vào nghiên cứu chính thức, có 2 nhân tố được chấp nhận có ảnh hưởng thuận chiều đến "cam kết tình cảm" là "tự chủ trong công việc" và "lợi ích". Trong đó, lợi ích nhân viên nhận được tác động mạnh đến cam kết tình cảm của họ hơn sự tự chủ trong công việc (hệ số hồi quy chuẩn hóa lớn hơn).

Thực hiện kiểm định tương quan giữa các biến 
Bảng 1. Kết quả kiểm định Cronbach's Alpha thang đo các nhân tố của mô hình nghiên cứu chính thức

\begin{tabular}{clccc}
\hline STT & Nhân tố & Số biến quan sát & $\begin{array}{c}\text { Hệ số tương } \\
\text { biến-tổng } \\
\text { quón } \\
\text { nhỏ nhất }\end{array}$ \\
\hline 1 & Sức khỏe và an toàn & 3 & 0,714 & 0,501 \\
2 & Cân bằng công việc và cuộc sống & 3 & 0,735 & 0,491 \\
3 & Đào tạo và phát triển & 5 & 0,925 & 0,780 \\
4 & Tự chủ trong công việc & 4 & 0,815 & 0,584 \\
5 & Lợi ích & 4 & 0,885 & 0,719 \\
6 & Cam kết tình cảm & 7 & 0,940 & 0,680 \\
7 & Cam kết duy trì & 4 & 0,891 & 0,6999 \\
\hline
\end{tabular}

Bảng 2. Kết quả phân tích hồi quy với biến phụ thuộc "cam kết tình cảm"

\begin{tabular}{lcccc}
\hline Biến & $\begin{array}{c}\text { Hệ số Beta } \\
\text { chuẩn hóa }\end{array}$ & Mức ý nghĩa $(P)$ & $\begin{array}{c}\text { Mức ý nghĩa trong } \\
\text { phân tích } \\
\text { Spearman }\end{array}$ \\
\hline Đào tạo và phát triển & 0,092 & 0,132 & 2,015 & 0,123 \\
Tự chủ trong công việc & 0,323 & 0,000 & 1,439 & 0,364 \\
Lợi ích & 0,540 & 0,000 & 1,659 & 0,151 \\
Sức khỏe và an toàn & 0,006 & 0,903 & 1,489 & 0,219 \\
Cân bằng công việc và cuộc sống & $-0,004$ & 0,947 & 1,710 & 0,066 \\
\hline R hiệu chỉnh & & 0,634 & 1,774 \\
Hệ số Durbin-Waston & & $<0,001$ & \\
Mức ý nghĩa $(P)$ & & \multicolumn{2}{c}{$<$} \\
\hline
\end{tabular}

Bảng 3. Bảng kết quả phân tích hồi quy của cam kết duy trì

\begin{tabular}{lcccc}
\hline Biến & $\begin{array}{c}\text { Hệ số Beta } \\
\text { chuẩn hóa }\end{array}$ & Mức ý nghĩa $(P)$ & $\begin{array}{c}\text { Mức ý nghĩa trong } \\
\text { phân tích } \\
\text { Spearman }\end{array}$ \\
\hline Đào tạo và phát triển & 0,229 & 0,010 & 2,015 & 0,323 \\
Tự chủ trong công việc & 0,158 & 0,034 & 1,439 & 0,457 \\
Lợi ích & 0,240 & 0,003 & 1,659 & 0,054 \\
Sức khỏe và an toàn & 0,005 & 0,952 & 1,489 & 0,656 \\
Cân bằng công việc và cuộc sống & 0,001 & 0,99 & 1,71 & 0,961 \\
\hline R hiệu chỉnh & & 0,243 & 1,886 \\
Hệ số Durbin-Waston & & $<0,001$ & \\
Mức ý nghĩa $(P)$ & & $<$ & \\
\hline
\end{tabular}

và phân tích hồi quy tuyến tính tác động của CSR đối với người lao động đến "cam kết duy trì" (Bảng 3) cho thấy mô hình xây dựng phù hợp. Mô hình cũng không bị hiện tượng tự tương quan (hệ số Durbin-Watson nằm trong khoảng từ 1 đến 3) (Ho, 2009), không xảy ra hiện tượng đa cộng tuyến (Hệ số phóng đại phương sai VIF < 3) và không vi phạm giả định phương sai không thay đổi (kết quả phân tích tương quan hạng Spearman giữa phần dư chuẩn hóa với các biến độc lập đều có giá trị $P>0,05)$.
Trong 5 nhân tố đưa vào nghiên cứu chính thức, ta chấp nhận 3 nhân tố là "đào tạo và phát triển", "tự chủ trong công việc" và "lợi ích" có ảnh hưởng thuận chiều đến cam kết tình cảm. Đồng thời, đối với "cam kết duy trì" của nhân viên công ty thì những lợi ích nhân viên nhận được vẫn có tác động mạnh nhất, kế đến là chính sách đào tạo và sự tự chủ trong công viêc.

Kết quả nghiên cứu phù hợp với kết quả của những nghiên cứu trước đây, cho thấy có sự ảnh hưởng tích cực của CSR đối với người lao động 
đến "cam kết tổ chức". Tuy nhiên, các nghiên cứu trước đây không đi sâu vào tác động của các nhân tố CSR đối với người lao động đến từng nhân tố của cam kết tổ chức.

Nghiên cứu cho thấy tại thị trường Việt Nam các nhân tố "tự chủ trong công việc" và "lợi ích" tác động đến "cam kết tình cảm". "Cam kết tình cảm" thể hiện sự gắn bó về mặt tình cảm của nhân viên đối với tổ chức của mình, mặc khác như kết quả phân tích đã thể hiện bên trên, khái niệm "cam kết tình cảm" trong nghiên cứu này còn thể hiện sự cam kết về mặt đạo đức, nhận thức của cá nhân về sự đúng đắn khi gắn bó với tổ chức. Vì vậy, mối liên hệ của "tự chủ trong công việc" và "lợi ích" dến "cam kết tình cảm" là hoàn toàn phù hợp. Nhân viên là con người và có những cảm xúc về cách họ được đối xử, khi nhân viên được tự chủ trong công việc, tức là được giao quyền để quyết định những vấn đề liên quan đến công việc của mình, họ cảm thấy thoải mái, có động lực để thực hiện nhiệm vụ vì chính họ mới là người hiểu rõ công việc của mình nhất, điều này dẫn đến hiệu suất làm việc tốt hơn, làm gia tăng sự hài lòng trong công việc và tất nhiên sẽ tăng tình cảm của họ với tổ chức. Bên cạnh đó, việc tự chủ trong công việc làm nhân viên cảm thấy kết quả công việc đạt được là thành quả của mình, họ sẽ ra sức bảo vệ, vun đắp và gắn bó. Mặt khác, những lợi ích về mặt kinh tế rõ ràng tác động đến cam kết của nhân viên với tổ chức, một chế độ đãi ngộ tốt, mức lương công bằng, phúc lợi đầy đủ luôn làm nhân viên cảm thấy những đóng góp của mình được công ty ghi nhận và đền bù xứng đáng, điều này làm gia tăng tình cảm cũng như trách nhiệm của họ, nhân viên cảm thấy cần phấn đấu và tiếp tục gắn bó với công ty.

So với "cam kết tình cảm" thì "cam kết duy trì" chịu thêm tác động từ biến "đào tạo và phát triển". "Cam kết duy trì" đề cập đến sự cam kết dựa trên tính toán, cân nhắc về lợi ích, chi phí giữa việc tiếp tục hay từ bỏ công việc hiện tại. Những lợi ích, chi phí này bao gồm những mất mát về vật chất, thời gian, vị trí,... Trong đó, một chương trình đào tạo tốt giúp nhân viên nâng cao những kỹ năng rõ ràng tác động nhiều đến quyết định của nhân viên khi họ nghĩ đến việc rời khỏi tổ chức. "Đào tạo và phát triển" cũng bao gồm cả cơ hội thăng tiến trong công việc, một công việc có thể không có những đãi ngộ hấp dẫn hiện tại nhưng mở ra cơ hội thăng tiến tốt cũng là một cách thức tăng "cam kết duy trì" nhờ những kỳ vọng về lợi ích trong tương lai.
Các nhân tố "sức khỏe và an toàn", "cân bằng công việc và cuộc sống" lại không khẳng định được ảnh hưởng đến cam kết tổ chức tại thị trường Việt Nam. Tuy là hai khái niệm khác nhau nhưng nhìn chung "sức khỏe và an toàn" và "cân bằng công việc và cuộc sống" đều đề cập đến vấn dề về sức khỏe của người lao động về thể chất lẫn tinh thần. Trong nền kinh tế đang phát triển, đời sống còn nhiều khó khăn như ở Việt Nam thì ý thức về sức khỏe lao động chưa được quan tâm lắm, phần lớn người lao động Việt xem trọng những lợi ích cụ thể, rõ ràng hơn. Mẫu điều tra với đa số là những nhân viên trẻ tuổi (số người dưới 30 tuổi chiếm $69 \%$ ) cũng có thể ảnh hưởng khá nhiều đến kết quả. Những người trẻ tuổi thường không chú ý nhiều đến sức khỏe cũng như cân bằng giữa công việc và cuộc sống. Nhiều người Việt hiện nay thích làm việc nhiều tiền thay vì muốn cống hiến cho công việc, nhất là giới trẻ. Trong một cuộc khảo sát thì $60 \%$ sinh viên chọn mục "Một công việc có thu nhập cao", chỉ 30\% chọn "Một công việc phù hợp với bản thân" (Mai, 2013). Điều hoàn hoàn toàn phù hợp với kết quả nghiên cứu khi nhân tố "lợi ích" thể hiện sự tác dộng lớn nhất trong cả hai nhân tố của "cam kết tổ chức" là "cam kết tình cảm" và "cam kết duy trì".

\section{Kết Luận}

Qua nghiên cứu có thể thấy rằng, việc thực hiện CSR là hết sức quan trọng, đặc biệt là trách nhiệm đối với đội ngũ nhân viên. Nghiên cứu đã cho thấy tác động tích cực của việc thực hiện CSR đối với nhân viên đến "cam kết tổ chức" của họ. Các doanh nghiệp cần thay dổi nhận thức của mình về việc thực hiện CSR, đây không chỉ là trách nhiệm mà còn là quyền lợi, nó mang lại những lợi ích thực tế cho doanh nghiệp. Doanh nghiệp cần xây dựng một chương trình chính sách trách nhiệm xã hội thống nhất từ lãnh đạo cao nhất đến từng nhân viên trong doanh nghiệp.

Kết quả nghiên cứu cũng cho thấy có sự khác biệt về cách nhìn nhận, đánh giá của người lao động Việt Nam so với các thị trường khác. Trong nhận thức của người lao động Việt Nam, chỉ khi có tình cảm với công ty họ mới cảm thấy có trách nhiệm và hợp đạo đức khi tiếp tục gắn bó lâu dài với công ty. Điều này cũng gợi ý cho các doanh nghiệp cần chú ý hơn đến các biện pháp giúp gia tăng tình cảm của nhân viên với tổ chức.

Một vấn đề khác là trong các nhân tố CSR với 
người lao động bên cạnh "lợi ích" thì yếu tố "tự chủ trong công việc" được đánh giá khá cao, điều này cho thấy người lao động ở nước ta hiện nay bắt đầu quan tâm nhiều hơn đến "cái tôi" của mình, họ muốn được tự do thể hiện năng lực bản thân, có chính kiến riêng chứ không đơn thuần là thực hiện những yêu cầu sẵn có của quản lý. Bất cứ ai cũng mong muốn được làm việc một cách thoải mái, chủ động hơn là làm theo những sự sắp đặt của người khác. Vì vậy, các công ty cần tạo cho nhân viên của mình sự tự chủ nhất định trong công việc của họ.

Tuy nhiên, "lợi ích" vẫn là một vấn đề cơ bản, có ảnh hưởng lớn nhất đến "cam kết tổ chức". "Lợi ích" ở đây không chỉ đơn thuần là tiền lương mà còn bao gồm những khoản phúc lợi khác như những khoản trợ cấp về $\mathrm{y}$ tế, giáo dục, chính sách hỗ trợ cho nhân viên cũng như các thành viên trong gia đình họ,... Một điểm quan trọng cần lưu ý là mấu chốt nằm ở sự công bằng. Công ty cần thể hiện cho nhân viên cảm nhận họ đang nhận được những gì xứng đáng với công sức của mình, những đóng góp của mình luôn được công ty ghi nhận.

Và cuối cùng một yếu tố nữa có ảnh hưởng đến cam kết tổ chức theo kết quả nghiên cứu là "đào tạo và phát triển". Các công ty cần xây dựng cho mình một chương trình đào tạo bài bản và tạo những cơ hội phát triển cho nhân viên của mình. Một khi được đào tạo phù hợp nhân viên dễ dàng hơn trong việc đáp ứng những yêu cầu của công việc, từ đó có những đóng góp tích cực cho sự thành công của công ty. Điều này cùng với một chế độ động viên, cơ hội thăng tiến hợp lý sẽ tác động đến tâm lý của họ, làm họ cảm thây gắn bó hơn, tạo nên sự cam kết mạnh mẽ.

\section{Tài Liệu Tham Khảo (References)}

Ali, I., Rehman, K., Yilmaz, A., Nazir, S., \& Ali, I. (2010). Effects of corporate social responsibility on consumer retention in cellular industry of Pakistan. African Journal of Business Management 4(4), 475485 .

Agarwal, R., \& Ferratt, T. W. (1999). Coping with labour scarcity in IT: strategies and practices for effective recruitment and retention. Cincinnati, USA: Pinnaflex.

Brammer, S., Millington, A., \& Rayton, B. (2007). The contribution of corporate social responsibility to organizational commitment. The International Journal of Human Resource Management 18(10), 1701-1719.

Carroll, A. B. (1999). Corporate social responsibility: evolution of a definitional construct. Business \& Society 38(3), 268-295.
Carroll, A. B. (1979). A three-dimensional conceptual model of corporate performance. Academy of Management Review 4(4), 497-505.

Chang, E. (1999). Career commitment as a complex moderator of organizational commitment and turnover intention. Human Relations 52(10), 1257-1278.

Dockel, A., Basson, J. S., \& Coetzee, M. (2006). The effect of retention factors on organisational commitment: an investigation of high technology employees. SA Journal of Human Resource Management 4(2), 2028.

Ho, S. M. (2009). Measurement of service quality and customer satisfaction and loyalty for ADSL service (Unpublished master's thesis). University of Economics Ho Chi Minh City, Ho Chi Minh City, Vietnam.

Jaros, S. (2007). Meyer and Allen model of organizational commitment: measurement issues. The Icfai Journal of Organizational Behavior 6(4), 7-25.

Le, H. T. (2006). Corporate social responsibility in salaries. Labor \&s Social Magazine 290, 20-23

Mai, C. (2013). Unemployed but still ... haughty! Labor Newspaper. Retrieved December 05, 2019, from http://nld.com.vn/cong-doan/that-nghiep-nhungvan-chanh-20131021064211666.htm.

Mathieu, J. E., \& Zajac, D. M. (1990). A review and meta-analysis of the antecedents, correlates, and consequences of organizational commitment. Psychological Bulletin 108(2), 171-194.

Meyer, J. P., Becker, T. E., \& Vandenberghe, C. (2004). Employee commitment and motivation: a conceptual analysis and integrative model. Journal of Applied Psychology 89(6), 991-1007.

Meyer, J. P., \& Allen, N. J. (1997). Commitment in the workplace: theory, research and application. California, USA: SAGE Publications.

Meyer, J. P., \& Allen, N. J. (1991). A three-component conceptualization of organizational commitment. $\mathrm{Hu}$ man resource Management Review 1(1), 61-89.

Nguyen, C. D., \& Luu, D. M. (2008). Corporate social responsibility: some theoretical issues and innovation requirements in state management in Vietnam. Economic Management Review 23, 3-11.

Nguyen, T. N. (2010). Linking human resource management and corporate social responsibility. VNU Journal of Science: Economics and Business 26, 232-238.

Pham, H. T. T. (2013). Evaluation of social responsibility index of enterprises applied at 23/9 Textile and Garment Joint Stock Company (Unpublished master's thesis). Da Nang University, Da Nang, Vietnam. 